\title{
Determinantal forms and recursive relations of the Delannoy two-functional sequence
}

\author{
Feng $\mathrm{Q}^{\mathrm{a}}$, Muhammet Cihat Dağ|l ${ }^{\mathrm{b}}$, Wei-Shih $\mathrm{Du}^{\mathrm{c}}$ \\ ${ }^{a}$ College of Mathematics and Physics, Inner Mongolia University for Nationalities, Tongliao 028043, Inner Mongolia, China; \\ School of Mathematical Sciences, Tianjin Polytechnic University, Tianjin 300387, China; \\ Institute of Mathematics, Henan Polytechnic University, Jiaozuo 454010, Henan, China. \\ ${ }^{b}$ Department of Mathematics, Akdeniz University, 07058-Antalya, Turkey. \\ ${ }^{c}$ Department of Mathematics, National Kaohsiung Normal University, Kaohsiung 82444, Taiwan.
}

\begin{abstract}
In the paper, the authors establish closed forms for the Delannoy two-functional sequence and its difference in terms of the Hessenberg determinants, derive recursive relations for the Delannoy two-functional sequence and its difference, and deduce closed forms in terms of the Hessenberg determinants and recursive relations for the Delannoy one-functional sequence, the Delannoy numbers, and central Delannoy numbers.

Keywords: closed form; recursive relation; difference; Hessenberg determinant; Delannoy two-functional sequence; Delannoy one-functional sequence; Delannoy number; central Delannoy number.

2010 MSC: 05A10, 11B83, 11C20, 11Y55, 26C05.

\section{Introduction}

A tridiagonal determinant is a determinant whose nonzero elements locate only on the diagonal and slots horizontally or vertically adjacent the diagonal. Technically speaking, a determinant $H=\left|h_{i j}\right|_{n \times n}$ is called a tridiagonal determinant if $h_{i j}=0$ for all pairs $(i, j)$ such that $|i-j|>1$. For more information, please refer to the paper [11]. A determinant $H=\left|h_{i j}\right|_{n \times n}$ is called a lower (or an upper, respectively) Hessenberg determinant if $h_{i j}=0$ for all pairs $(i, j)$ such that $i+1<j$ (or $j+1<i$, respectively). For more information, please refer to the paper [18].
\end{abstract}

Email addresses: qifeng618@gmail.com, qifeng618@hotmail.com, qifeng618@qq.com (Feng Qi), mcihatdagli@akdeniz.edu.tr (Muhammet Cihat Dağlı), wsdu@mail.nknu.edu.tw (Wei-Shih Du) 
In combinatorial number theory, the Delannoy number $D(m, n)$ for $n, m \geq 0$ can be regarded as the number of lattice paths from $(0,0)$ to $(m, n)$ in which only east $(1,0)$, north $(0,1)$, and northeast $(1,1)$. The Delannoy numbers $D(m, n)$ can be defined by means of the recurrence relation

$$
D(m, n)=D(m-1, n)+D(m-1, n-1)+D(m, n-1)
$$

with initial values $D(0, n)=D(m, 0)=D(0,0)=1$. The historic significance of these numbers $D(m, n)$ was explained in the paper [1]. The Delannoy numbers $D(m, n)$ can be computed by explicit formulas

$$
D(m, n)=\sum_{k=0}^{n}\left(\begin{array}{l}
n \\
k
\end{array}\right)\left(\begin{array}{l}
m \\
k
\end{array}\right) 2^{k} \quad \text { and } \quad D(m, n)=\sum_{\ell=0}^{n}\left(\begin{array}{c}
n \\
\ell
\end{array}\right)\left(\begin{array}{c}
m+n-\ell \\
n
\end{array}\right)
$$

and can be generated by

$$
\frac{1}{1-x-y-x y}=\sum_{n, m \geq 0} D(m, n) x^{m} y^{n} .
$$

For more information on the Delannoy numbers $D(m, n)$, please refer to [1, 19, 59, 63] and closely related references therein.

The numbers $D(k)=D(k, k)$ for $k \geq 0$ are known as central Delannoy numbers. In [26, Theorem 1.3], central Delannoy numbers $D(k)$ were represented by

$$
D(k)=\frac{1}{\pi} \int_{3-2 \sqrt{2}}^{3+2 \sqrt{2}} \frac{1}{\sqrt{(t-3+2 \sqrt{2})(3+2 \sqrt{2}-t)}} \frac{1}{t^{k+1}} \mathrm{~d} t, \quad k \geq 0 .
$$

In [48, Section 4], the central Delannoy numbers $D(k)$ were generalized as

$$
D_{a, b}(k)=\frac{1}{\pi} \int_{a}^{b} \frac{1}{\sqrt{(t-a)(b-t)}} \frac{1}{t^{k+1}} \mathrm{~d} t, \quad k \geq 0, \quad b>a>0
$$

and, from [26, Lemma 2.4], it is derived that the quantities $D_{a, b}(k)$ have the generating function

$$
\frac{1}{\sqrt{(x+a)(x+b)}}=\sum_{k=0}^{\infty} D_{a, b}(k) x^{k}
$$

By virtue of conclusions in [24, Section 2.4] and [38, Remark 4.1], we can find that the quantities $D_{a, b}(k)$ for $k \geq 0$ can be computed by

$$
D_{a, b}(k)=\frac{1}{G(a, b)} \frac{(-1)^{k}}{[2 A(a, b)]^{k}} \sum_{\ell=0}^{k}(-1)^{\ell} 2^{2 \ell} \frac{(2 \ell-1) ! !}{(2 \ell) ! !}\left(\begin{array}{c}
\ell \\
k-\ell
\end{array}\right)\left[\frac{A(a, b)}{H(a, b)}\right]^{\ell}
$$

and

$$
D_{a, b}(k)=\frac{1}{\sqrt{a b}} \frac{1}{b^{k}} \sum_{\ell=0}^{k} \frac{(2 \ell-1) ! !}{(2 \ell) ! !} \frac{[2(k-\ell)-1] ! !}{[2(k-\ell)] ! !}\left(\frac{b}{a}\right)^{\ell},
$$

where $A(a, b)=\frac{a+b}{2}, G(a, b)=\sqrt{a b}$, and $H(a, b)=\frac{2}{1 / a+1 / b}$ are respectively known as arithmetic, geometric, and harmonic means of $a, b>0$. The quantities $D_{a, b}(k)$ can be further generalized as

$$
D_{a, b ; \lambda}(z)=\frac{\sin (\lambda \pi)}{\pi} \int_{a}^{b} \frac{1}{(t-a)^{\lambda}(b-t)^{1-\lambda}} \frac{1}{t^{z+1}} \mathrm{~d} t,
$$

where $0<a<b, \lambda \in(0,1)$, and $z \in \mathbb{C}$. It is obvious that $D_{a, b ; 1 / 2}(k)=D_{a, b}(k)$. Theorems 2.1 and 2.2 in the paper [38] demonstrate that the quantities $D_{a, b ; \lambda}(k)$ can be generated by

$$
\frac{1}{(x+a)^{\lambda}(x+b)^{1-\lambda}}=\sum_{k=0}^{\infty} D_{a, b ; \lambda}(k) x^{k}
$$


and can be computed by the closed forms

$$
D_{a, b ; \lambda}(k)=\frac{1}{n !} \frac{1}{a^{\lambda} b^{1-\lambda}} \frac{1}{b^{n}} \sum_{\ell=0}^{n}\left(\begin{array}{l}
n \\
\ell
\end{array}\right)(\lambda)_{\ell}(1-\lambda)_{n-\ell}\left(\frac{b}{a}\right)^{\ell}
$$

and

$$
D_{a, b ; \lambda}(k)=\frac{1}{a^{\lambda} b^{1-\lambda}} \frac{1}{b^{n}} \sum_{k=0}^{n}\left(\frac{b}{a}\right)^{k} \sum_{\ell=0}^{k}\langle\lambda\rangle_{\ell}\left(\begin{array}{c}
k-1 \\
\ell-1
\end{array}\right) \frac{1}{\ell !}\left(1-\frac{a}{b}\right)^{\ell},
$$

where

$$
(x)_{n}= \begin{cases}\prod_{k=0}^{n-1}(x+k), & n \geq 1 \\ 1, & n=0\end{cases}
$$

and

$$
\langle x\rangle_{n}= \begin{cases}\prod_{k=0}^{n-1}(x-k), & n \geq 1 \\ 1, & n=0\end{cases}
$$

are known as the rising and falling factorials respectively. The ideas, significance, and reasonability of these generalizations $D_{a, b}(k)$ and $D_{a, b ; \lambda}(z)$ come from the papers [25, 34, 37, 38, 39, 43, 52, 53, 54, 55] and closely related references therein.

Inspired by the identity

$$
\sum_{k=0}^{n}\left(\begin{array}{l}
n \\
k
\end{array}\right)\left(\begin{array}{l}
x \\
k
\end{array}\right) t^{k}=\sum_{k=0}^{n}\left(\begin{array}{l}
n \\
k
\end{array}\right)\left(\begin{array}{c}
x+k \\
n
\end{array}\right)(t-1)^{k}
$$

in [8, eq. 3.17], the Delannoy numbers $D(m, n)$ were extended to the functional sequence

$$
D(x ; n)=\sum_{k=0}^{n}\left(\begin{array}{l}
n \\
k
\end{array}\right)\left(\begin{array}{l}
x \\
k
\end{array}\right) 2^{k}=\sum_{k=0}^{n}\left(\begin{array}{l}
n \\
k
\end{array}\right)\left(\begin{array}{c}
x+k \\
n
\end{array}\right)
$$

in [61]. Various arithmetic properties and congruence relations for the Delannoy one-functional sequence $D(x ; n)$ have been studied in [9, 16, 17, 59, 60, 63.

In [58], the Delannoy one-functional sequence $D(x ; n)$ was further generalized as

$$
D(x, r ; n)=\sum_{k=0}^{n}\left(\begin{array}{c}
x+r+k \\
k
\end{array}\right)\left(\begin{array}{l}
x-r \\
n-k
\end{array}\right),
$$

the generating function

$$
\frac{(1+t)^{x-r}}{(1-t)^{x+r+1}}=\sum_{n=0}^{\infty} D(x, r ; n) t^{n}, \quad|t|<1
$$

was derived, and a plenty of identities for the Delannoy two-functional sequence $D(x, r ; n)$ were acquired.

In [7, the Delannoy two-functional sequence $D(x, r ; n)$ was generalized again to the Delannoy twofunctional polynomials

$$
D(x, r ; n ; y)=\sum_{k=0}^{n}\left(\begin{array}{c}
x+r+k \\
k
\end{array}\right)\left(\begin{array}{l}
x-r \\
n-k
\end{array}\right) y^{k}
$$

and, among other things, the generating function and recurrence formula for the Delannoy two-functional polynomials $D(x, r ; n ; y)$ were derived.

It is noted that $D(x, 0 ; n)=D(x ; n)$ and $D(x, r ; n ; 1)=D(x, r ; n)$. 
In combinatorial number theory, it is significant to express concrete sequences or arrays of integer numbers or polynomials in terms of tridiagonal determinants or the Hessenberg determinants. In this respect, the Bernoulli numbers and polynomials [2, 6, 20, 27, 36, 40, 62, the Euler numbers and polynomials [29, 32, 64], (central) Delannoy numbers and polynomials [10, 19, 25, 26, 31, 47, 48, 49], the Horadam polynomials [41], (generalized) Fibonacci numbers and polynomials [13, 14, 15, 21, 25, 33, 46, 50, the Lucas polynomials [41, [46], and the like, have been represented via tridiagonal determinants or the Hessenberg determinants, and consequently many remarkable relations have been obtained. For more information in this area and direction, please refer to [12, 22, 23, 30, 35, 42, 44, 45, 51, 56, 57] and closely related references therein.

In this paper, we will present closed forms [3] for the Delannoy two-functional sequence $D(x, r ; n)$ and its difference $D(x, r ; n)-D(x, r ; n-1)$ in terms of the Hessenberg determinants, derive recursive relations for the Delannoy two-functional sequence $D(x, r ; n)$ and its difference $D(x, r ; n)-D(x, r ; n-1)$, and deduce closed forms in terms of the Hessenberg determinants and recursive relations for the Delannoy one-functional sequence $D(x ; n)$, the Delannoy numbers $D(m, n)$, and central Delannoy numbers $D(k)$.

\section{Determinantal forms of the Delannoy two-functional sequence and its difference}

In this section, we will present closed forms for the Delannoy two-functional sequence $D(x, r ; n)$ and for its difference $D(x, r ; n)-D(x, r ; n-1)$ in terms of the Hessenberg determinants.

Theorem 2.1. The Delannoy two-functional sequence $D(x, r ; n)$ for $n \geq 0$ can be determinantally expressed by

$$
D(x, r ; n)=\frac{(-1)^{n}}{n !}\left|P_{(n+1) \times 1}(x, r) \quad B_{(n+1) \times n}(x, r)\right|_{(n+1) \times(n+1)},
$$

where

$$
\begin{aligned}
& P_{(n+1) \times 1}(x, r)=\left(\begin{array}{lllll}
\langle x-r\rangle_{0} & \langle x-r\rangle_{1} & \langle x-r\rangle_{2} & \ldots & \langle x-r\rangle_{n}
\end{array}\right)^{T}, \\
& B_{(n+1) \times n}(x, r)=\left(\left(\begin{array}{c}
i-1 \\
j-1
\end{array}\right)(-1)^{i-j}\langle x+r+1\rangle_{i-j}\right)_{(n+1) \times n},
\end{aligned}
$$

and $T$ denotes the transpose of a matrix.

The difference $D(x, r ; n)-D(x, r ; n-1)$ for $n \geq 1$ can be determinantally expressed by

$$
D(x, r ; n)-D(x, r ; n-1)=\frac{(-1)^{n}}{n !}\left|P_{(n+1) \times 1} \quad C_{(n+1) \times n}\right|_{(n+1) \times(n+1)},
$$

where

$$
C_{(n+1) \times n}(x, r)=\left(\left(\begin{array}{c}
i-1 \\
j-1
\end{array}\right)(-1)^{i-j}\langle x+r\rangle_{i-j}\right)_{(n+1) \times n} .
$$

Proof. Let $p_{x, r}(t)=(1+t)^{x-r}$ and $q_{x, r}(t)=(1-t)^{x+r+1}$. Then

$$
p_{x, r}^{(k)}(t)=\langle x-r\rangle_{k}(1+t)^{x-r-k} \rightarrow\langle x-r\rangle_{k}
$$

and

$$
q_{x, r}^{(k)}(t)=(-1)^{k}\langle x+r+1\rangle_{k}(1-t)^{x-r-k} \rightarrow(-1)^{k}\langle x+r+1\rangle_{k}
$$

as $t \rightarrow 0$.

The equation (1.1) implies that

$$
D(x, r ; n)=\frac{1}{n !} \lim _{t \rightarrow 0} \frac{\mathrm{d}^{n}}{\mathrm{~d} t^{n}}\left[\frac{(1+t)^{x-r}}{(1-t)^{x+r+1}}\right] .
$$

Let $u(t)$ and $v(t) \neq 0$ be two $n$th differentiable functions for $n \in \mathbb{N}$. Exercise 5$)$ in [4, p. 40] reads that the $n$th derivative of the ratio $\frac{u(t)}{v(t)}$ can be computed by

$$
\frac{\mathrm{d}^{n}}{\mathrm{~d} x^{n}}\left[\frac{u(t)}{v(t)}\right]=(-1)^{n} \frac{\left|W_{(n+1) \times(n+1)}(t)\right|}{v^{n+1}(t)},
$$


where $\left|W_{(n+1) \times(n+1)}(t)\right|$ is the determinant of the $(n+1) \times(n+1)$ matrix

$$
W_{(n+1) \times(n+1)}(t)=\left(U_{(n+1) \times 1}(t) \quad V_{(n+1) \times n}(t)\right)_{(n+1) \times(n+1)},
$$

the matrix $U_{(n+1) \times 1}(t)$ is an $(n+1) \times 1$ matrix whose elements satisfy $u_{k, 1}(t)=u^{(k-1)}(t)$ for $1 \leq k \leq n+1$, and $V_{(n+1) \times n}(t)$ is an $(n+1) \times n$ matrix whose elements meet $v_{i, j}(t)=\left(\begin{array}{c}i-1 \\ j-1\end{array}\right) v^{(i-j)}(t)$ for $1 \leq i \leq n+1$ and $1 \leq j \leq n$. The formula (2.4) is a general and fundamental, but non-extensively circulated, formula for derivatives of a ratio of two differentiable functions.

Combining (2.3) and (2.4) gives

$$
\begin{aligned}
D(x, r ; n) & =\frac{(-1)^{n}}{n !} \lim _{t \rightarrow 0} \frac{\left|U_{(n+1) \times 1}(t) V_{(n+1) \times n}(t)\right|}{q_{x, r}^{n+1}(t)} \\
& =\frac{(-1)^{n}}{n !} \frac{\left|\lim _{t \rightarrow 0} U_{(n+1) \times 1}(t) \lim _{t \rightarrow 0} V_{(n+1) \times n}(t)\right|}{\lim _{t \rightarrow 0} q_{x, r}^{n+1}(t)} \\
& =\frac{(-1)^{n}}{n !}\left|\lim _{t \rightarrow 0} U_{(n+1) \times 1}(t) \quad \lim _{t \rightarrow 0} V_{(n+1) \times n}(t)\right|,
\end{aligned}
$$

where

$$
\begin{aligned}
\lim _{t \rightarrow 0} U_{(n+1) \times 1}(t) & =\lim _{t \rightarrow 0}\left(\begin{array}{lllll}
p_{x, r}^{(0)}(t) & p_{x, r}^{(1)}(t) & p_{x, r}^{(2)}(t) & \ldots & p_{x, r}^{(n)}(t)
\end{array}\right)^{T} \\
& =\left(\begin{array}{llllll}
\langle x-r\rangle_{0} & \langle x-r\rangle_{1} & \langle x-r\rangle_{2} & \ldots & \langle x-r\rangle_{n}
\end{array}\right)^{T}
\end{aligned}
$$

and

$$
\lim _{t \rightarrow 0} V_{(n+1) \times n}(t)=\left(\lim _{t \rightarrow 0}\left(\begin{array}{c}
i-1 \\
j-1
\end{array}\right) q_{x, r}^{(i-j)}(t)\right)_{(n+1) \times n}=\left(\left(\begin{array}{c}
i-1 \\
j-1
\end{array}\right)(-1)^{i-j}\langle x+r+1\rangle_{i-j}\right)_{(n+1) \times n} .
$$

The determinantal form $(2.1)$ is thus proved.

The generating function in (1.1) can be rewritten as

$$
\frac{(1+t)^{x-r}}{(1-t)^{x+r}}=(1-t) \sum_{n=0}^{\infty} D(x, r ; n) t^{n}=D(x, r ; 0)+\sum_{n=1}^{\infty}[D(x, r ; n)-D(x, r ; n-1)] t^{n} .
$$

By the same arguments as in the derivation of the determinantal form 2.1), we can obtain the determinantal forms (2.2) immediately. The proof of Theorem 2.1 is complete.

\section{Recursive relations of the Delannoy two-functional sequence and its difference}

In this section, with the aid of Theorem 2.1, we will derive recursive relations for the Delannoy twofunctional sequence $D(x, r ; n)$ and for its differences $D(x, r ; n)-D(x, r ; n-1)$.

Theorem 3.1. The Delannoy two-functional sequence $D(x, r ; n)$ for $n \geq 1$ satisfies

$$
D(x, r ; n)=\frac{\langle x-r\rangle_{n}}{n !}+(-1)^{n} \sum_{s=1}^{n}(-1)^{s} \frac{\langle x+r+1\rangle_{n-s+1}}{(n-s+1) !} D(x, r ; s-1)
$$

and the differences $D(x, r ; n)-D(x, r ; n-1)$ for $n \geq 2$ meet

$$
D(x, r ; n)-D(x, r ; n-1)=\frac{\langle x-r\rangle_{n}}{n !}+(-1)^{n} \sum_{s=2}^{n}(-1)^{s} \frac{\langle x+r\rangle_{n-s+1}}{(n-s+1) !}[D(x, r ; s-1)-D(x, r ; s-2)] .
$$


Proof. Let $Q_{0}=1$ and

$$
Q_{n}=\left|\begin{array}{cccccc}
e_{1,1} & e_{1,2} & 0 & \ldots & 0 & 0 \\
e_{2,1} & e_{2,2} & e_{2,3} & \ldots & 0 & 0 \\
e_{3,1} & e_{3,2} & e_{3,3} & \ldots & 0 & 0 \\
\vdots & \vdots & \vdots & \vdots & \vdots & \vdots \\
e_{n-2,1} & e_{n-2,2} & e_{n-2,3} & \ldots & e_{n-2, n-1} & 0 \\
e_{n-1,1} & e_{n-1,2} & e_{n-1,3} & \ldots & e_{n-1, n-1} & e_{n-1, n} \\
e_{n, 1} & e_{n, 2} & e_{n, 3} & \ldots & e_{n, n-1} & e_{n, n}
\end{array}\right|
$$

for $n \in \mathbb{N}$. In [5, p. 222, Theorem], it was proved that the sequence $Q_{n}$ for $n \geq 0$ satisfies $Q_{1}=e_{1,1}$ and

$$
Q_{n}=\sum_{s=1}^{n}(-1)^{n-s} e_{n, s}\left(\prod_{j=s}^{n-1} e_{j, j+1}\right) Q_{s-1}
$$

for $n \geq 2$, where any empty product is understood to be 1 while any empty sum is understood to be 0 .

Substituting $(-1)^{n-1}(n-1) ! D(x, r ; n-1)$ for $Q_{n}$, replacing $e_{k, 1}$ for $1 \leq k \leq n$ by $\langle x-r\rangle_{k-1}$, switching $e_{i, j}$ for $1 \leq i \leq n$ and $2 \leq j \leq n$ into $\left(\begin{array}{l}i-1 \\ j-2\end{array}\right)(-1)^{i-j+1}\langle x+r+1\rangle_{i-j+1}$ in (3.3), and simplifying arrive at

$$
\begin{aligned}
& (-1)^{n-1}(n-1) ! D(x, r ; n-1) \\
& \quad=(-1)^{n-1}\langle x-r\rangle_{n-1}+\sum_{s=2}^{n}(-1)^{n-s}\left(\begin{array}{c}
n-1 \\
s-2
\end{array}\right)(-1)^{n-s+1}\langle x+r+1\rangle_{n-s+1}(-1)^{s-2}(s-2) ! D(x, r ; s-2)
\end{aligned}
$$

which can be reformulated as

$$
\begin{aligned}
&(-1)^{n-1}(n-1) ! D(x, r ; n-1) \\
& \quad=(-1)^{n-1}\langle x-r\rangle_{n-1}-\sum_{s=2}^{n}(-1)^{s}(s-2) !\left(\begin{array}{c}
n-1 \\
s-2
\end{array}\right)\langle x+r+1\rangle_{n-s+1} D(x, r ; s-2) .
\end{aligned}
$$

The recursive relation $(3.1)$ is thus proved.

Similarly, substituting $(-1)^{n-1}(n-1) ![D(x, r ; n-1)-D(x, r ; n-2)]$ for $Q_{n}$, replacing $e_{k, 1}$ for $1 \leq k \leq n$ by $\langle x-r\rangle_{k-1}$, switching $e_{i, j}$ for $1 \leq i \leq n$ and $2 \leq j \leq n$ into $\left(\begin{array}{c}i-1 \\ j-2\end{array}\right)(-1)^{i-j+1}\langle x+r\rangle_{i-j+1}$ in $(3.3)$, and straightforwardly simplifying lead to the recursive relation 3.2 . The proof of Theorem 3.1 is complete.

\section{Determinantal forms and recursive relations for the Delannoy one-functional sequence, the Delannoy numbers, and central Delannoy numbers}

In this section, with the help of Theorems 2.1 and 3.1, we deduce closed forms in terms of the Hessenberg determinants and recursive relations for the Delannoy one-functional sequence $D(x ; n)$, the Delannoy numbers $D(m, n)$, and central Delannoy numbers $D(k)$.

Since $D(x, 0 ; n)=D(x ; n)$, when taking $r=0$ in Theorems 2.1 and 3.1 , we derive the following conclusions.

Theorem 4.1. The Delannoy one-functional sequence $D(x ; n)$ for $n \geq 0$ can be determinantally expressed by

$$
D(x ; n)=\frac{(-1)^{n}}{n !}\left|\mathfrak{P}_{(n+1) \times 1}(x) \quad \mathfrak{B}_{(n+1) \times n}(x)\right|_{(n+1) \times(n+1)},
$$

where

$$
\mathfrak{P}_{(n+1) \times 1}(x)=\left(\begin{array}{lllll}
\langle x\rangle_{0} & \langle x\rangle_{1} & \langle x\rangle_{2} & \ldots & \langle x\rangle_{n}
\end{array}\right)^{T}
$$


and

$$
\mathfrak{B}_{(n+1) \times n}(x)=\left(\left(\begin{array}{c}
i-1 \\
j-1
\end{array}\right)(-1)^{i-j}\langle x+1\rangle_{i-j}\right)_{(n+1) \times n} .
$$

The difference $D(x ; n)-D(x ; n-1)$ for $n \geq 1$ can be determinantally expressed by

$$
D(x ; n)-D(x ; n-1)=\frac{(-1)^{n}}{n !}\left|\mathfrak{P}_{(n+1) \times 1}(x) \quad \mathfrak{C}_{(n+1) \times n}(x)\right|_{(n+1) \times(n+1)},
$$

where

$$
\mathfrak{C}_{(n+1) \times n}(x)=\left(\left(\begin{array}{c}
i-1 \\
j-1
\end{array}\right)(-1)^{i-j}\langle x\rangle_{i-j}\right)_{(n+1) \times n} .
$$

Theorem 4.2. The Delannoy one-functional sequence $D(x ; n)$ for $n \geq 1$ satisfies

$$
D(x ; n)=\frac{\langle x\rangle_{n}}{n !}+(-1)^{n} \sum_{s=1}^{n}(-1)^{s} \frac{\langle x+1\rangle_{n-s+1}}{(n-s+1) !} D(x ; s-1)
$$

and the differences $D(x ; n)-D(x ; n-1)$ for $n \geq 2$ meet

$$
D(x ; n)-D(x ; n-1)=\frac{\langle x\rangle_{n}}{n !}+(-1)^{n} \sum_{s=2}^{n}(-1)^{s} \frac{\langle x\rangle_{n-s+1}}{(n-s+1) !}[D(x ; s-1)-D(x ; s-2)] .
$$

When taking $x=m$ in Theorems 4.3 and 4.4 , we derive the following conclusions.

Theorem 4.3. The Delannoy numbers $D(m ; n)$ for $m, n \geq 0$ can be determinantally expressed by

$$
D(m ; n)=\frac{(-1)^{n}}{n !}\left|\mathcal{P}_{(n+1) \times 1}(m) \quad \mathcal{B}_{(n+1) \times n}(m)\right|_{(n+1) \times(n+1)},
$$

where

and

$$
\mathcal{P}_{(n+1) \times 1}(m)=\left(\begin{array}{lllll}
\langle m\rangle_{0} & \langle m\rangle_{1} & \langle m\rangle_{2} & \ldots & \langle m\rangle_{n}
\end{array}\right)^{T}
$$

$$
\mathcal{B}_{(n+1) \times n}(m)=\left((-1)^{i-j}\left(\begin{array}{c}
i-1 \\
j-1
\end{array}\right)\langle m+1\rangle_{i-j}\right)_{(n+1) \times n} .
$$

The differences $D(m ; n)-D(m ; n-1)$ for $m \geq 0$ and $n \geq 1$ can be determinantally expressed by

$$
D(m ; n)-D(m ; n-1)=\frac{(-1)^{n}}{n !}\left|\mathcal{P}_{(n+1) \times 1}(m) \quad \mathcal{C}_{(n+1) \times n}(m)\right|_{(n+1) \times(n+1)},
$$

where

$$
\mathcal{C}_{(n+1) \times n}(m)=\left(\left(\begin{array}{c}
i-1 \\
j-1
\end{array}\right)(-1)^{i-j}\langle m\rangle_{i-j}\right)_{(n+1) \times n} .
$$

Theorem 4.4. The Delannoy numbers $D(m ; n)$ for $m \geq 0$ and $n \geq 1$ satisfy

$$
D(m ; n)=\left(\begin{array}{c}
m \\
n
\end{array}\right)+(-1)^{n-1} \sum_{s=0}^{n-1}(-1)^{s}\left(\begin{array}{c}
m+1 \\
n-s
\end{array}\right) D(m ; s)
$$

and the differences $D(m ; n)-D(m ; n-1)$ for $m \geq 0$ and $n \geq 2$ meet

$$
D(m ; n)-D(m ; n-1)=\left(\begin{array}{c}
m \\
n
\end{array}\right)+(-1)^{n} \sum_{s=2}^{n}(-1)^{s}\left(\begin{array}{c}
m \\
n-s+1
\end{array}\right)[D(m ; s-1)-D(m ; s-2)] .
$$

When taking $m=n=k$ in Theorem 4.3, we derive the following conclusions.

Theorem 4.5. Central Delannoy numbers $D(k)$ for $k \geq 0$ can be determinantally expressed by

$$
D(k)=\frac{(-1)^{k}}{k !}\left|\mathcal{P}_{(k+1) \times 1} \quad \mathcal{B}_{(k+1) \times k}\right|_{(k+1) \times(k+1)},
$$

where

and

$$
\mathcal{P}_{(k+1) \times 1}=\left(\begin{array}{lllll}
\langle k\rangle_{0} & \langle k\rangle_{1} & \langle k\rangle_{2} & \cdots & \langle k\rangle_{k}
\end{array}\right)^{T}
$$

$$
\mathcal{B}_{(k+1) \times k}=\left(\left(\begin{array}{c}
i-1 \\
j-1
\end{array}\right)(-1)^{i-j}\langle k+1\rangle_{i-j}\right)_{(k+1) \times k} .
$$




\section{Remarks}

Finally we list several remarks as follows.

1. The determinantal forms 4.2 and 4.4 in Theorem 4.3 and Theorem 4.5 recover [19, Theorem 2.1].

2. The formula (4.3) coincides with the first result in [19, Theorem 3.1]. Letting $m=n$ in 4.3 recovers the second result in [19, Theorem 3.1].

3. The determinantal form (4.4) in Theorem 4.5 is different from

$$
D(k)=(-1)^{k}\left|\begin{array}{ccccccc}
a_{1} & 1 & 0 & \cdots & 0 & 0 & 0 \\
a_{2} & a_{1} & 1 & \cdots & 0 & 0 & 0 \\
a_{3} & a_{2} & a_{1} & \cdots & 0 & 0 & 0 \\
\vdots & \vdots & \vdots & \ddots & \vdots & \vdots & \vdots \\
a_{k-2} & a_{k-3} & a_{k-4} & \cdots & a_{1} & 1 & 0 \\
a_{k-1} & a_{k-2} & a_{k-3} & \cdots & a_{2} & a_{1} & 1 \\
a_{k} & a_{k-1} & a_{k-2} & \cdots & a_{3} & a_{2} & a_{1}
\end{array}\right|
$$

where $k \geq 1$ and

$$
a_{k}=\frac{(-1)^{k+1}}{6^{k}} \sum_{\ell=1}^{k}(-1)^{\ell} 6^{2 \ell} \frac{(2 \ell-3) ! !}{(2 \ell) ! !}\left(\begin{array}{c}
\ell \\
k-\ell
\end{array}\right) .
$$

The determinantal form (5.1) was established in [26, Theorem 1.1]. See also related texts in the papers [19].

4. This paper is a modified version of the electronic preprint [28].

\section{Funding}

The third author is supported by a project (Grant No. MOST 107-2115-M-017-004-MY2) from the Ministry of Science and Technology of the Republic of China.

\section{Conflict of interest}

The authors declare that they have no conflict of interest.

\section{References}

[1] C. Banderier and S. Schwer, Why Delannoy numbers?, J. Statist. Plann. Inference 135 (2005), no. 1, 40-54.

[2] R. Booth and H. D. Nguyen, Bernoulli polynomials and Pascal's square, Fibonacci Quart. 46/47 (2008/2009), no. 1, 38-47.

[3] J. M. Borwein and R. E. Crandall, Closed forms: what they are and why we care, Notices Amer. Math. Soc. 60 (2013), no. $1,50-65$.

[4] N. Bourbaki, Functions of a Real Variable, Elementary Theory, Translated from the 1976 French original by Philip Spain. Elements of Mathematics (Berlin). Springer-Verlag, Berlin, 2004.

[5] N. D. Cahill, J. R. D’Errico, D. A. Narayan, and J. Y. Narayan, Fibonacci determinants, College Math. J. 33 (2002), no. 3, $221-225$.

[6] F. Costabile, F. Dell'Accio, M. I. Gualtieri, A new approach to Bernoulli polynomials, Rend. Mat. Appl. (7) 26 (2006), no. $1,1-12$.

[7] M. C. Dağlı, A new generalization of Delannoy numbers, accepted for publication in Indian Journal of Pure and Applied Mathematics.

[8] H. W. Gould, Combinatorial Identities: A standardized set of tables listing 500 binomial coefficient summations, Henry W. Gould, Morgantown, W.Va., 1972.

[9] V. J. W. Guo, Proof of Sun's conjectures on integer-valued polynomials, J. Math. Anal. Appl. 444 (2016), no. 1, $182-191$.

[10] B.-N. Guo and F. Qi, Some explicit and recursive formulas of the large and little Schröder numbers, Arab J. Math. Sci. 23 (2017), no. 2, 141-147.

[11] V. Higgins and C. Johnson, Inverse spectral problems for collections of leading principal submatrices of tridiagonal matrices, Linear Algebra Appl. 489 (2016), 104-122.

[12] S. Hu and M.-S. Kim, Two closed forms for the Apostol-Bernoulli polynomials, Ramanujan J. 46 (2018), no. 1, $103-117$. 
[13] A. İpek and K. Arı, On Hessenberg and pentadiagonal determinants related with Fibonacci and Fibonacci-like numbers, Appl. Math. Comput. 229 (2014), 433-439.

[14] M. Janjić, Determinants and recurrence sequences, J. Integer Seq. 15 (2012), no. 3, Article 12.3.5, 21 pages.

[15] R. K. Kittappa, A representation of the solution of the nth order linear difference equation with variable coefficients, Linear Algebra Appl. 193 (1993), 211-222.

[16] J.-C. Liu, A supercongruence involving Delannoy numbers and Schröder numbers, J. Number Theory 168 (2016), $117-127$.

[17] J.-C. Liu, L. Li, and S.-D. Wang, Some congruences on Delannoy numbers and Schröder numbers, Int. J. Number Theory 14 (2018), no. 7, 2035-2041.

[18] R. S. Martin and J. H. Wilkinson, Handbook Series Linear Algebra: Similarity reduction of a general matrix to Hessenberg form, Numer. Math. 12 (1968), no. 5, 349-368.

[19] F. Qi, A determinantal expression and a recursive relation of the Delannoy numbers, Acta Univ. Sapientiae Math. 12 (2020), no. 2, in press; arXiv preprint (2020), available online at https://arxiv.org/abs/2003.12572.

[20] F. Qi, A double inequality for the ratio of two non-zero neighbouring Bernoulli numbers, J. Comput. Appl. Math. 351 (2019), 1-5.

[21] F. Qi, Denying a short proof of a determinantal formula for generalized Fibonacci polynomials, J. Math. Anal. 11 (2020), no. $1,52-57$.

[22] F. Qi, Determinantal expressions and recurrence relations for Fubini and Eulerian polynomials, J. Interdiscip. Math. 22 (2019), no. 3, 317-335.

[23] F. Qi, Derivatives of tangent function and tangent numbers, Appl. Math. Comput. 268 (2015), 844-858.

[24] F. Qi and V. Čerňanová, Some discussions on a kind of improper integrals, Int. J. Anal. Appl. 11 (2016), no. 2, $101-109$.

[25] F. Qi, V. Čerňanová, and Y. S. Semenov, Some tridiagonal determinants related to central Delannoy numbers, the Chebyshev polynomials, and the Fibonacci polynomials, Politehn. Univ. Bucharest Sci. Bull. Ser. A Appl. Math. Phys. 81 (2019), no. 1, $123-136$.

[26] F. Qi, V. Čerňanová, X.-T. Shi, and B.-N. Guo, Some properties of central Delannoy numbers, J. Comput. Appl. Math. 328 (2018), 101-115.

[27] F. Qi and R. J. Chapman, Two closed forms for the Bernoulli polynomials, J. Number Theory 159 (2016), 89-100.

[28] F. Qi, M. C. Dağll, and W.-S. Du, Determinantal forms and recursive relations of the Delannoy two-functional sequence, OSF Preprints (2020), available online at https://doi.org/10.31219/osf.io/u683y

[29] F. Qi and B.-N. Guo, A determinantal expression and a recurrence relation for the Euler polynomials, Adv. Appl. Math. Sci. 16 (2017), no. 9, 297-309.

[30] F. Qi and B.-N. Guo, A diagonal recurrence relation for the Stirling numbers of the first kind, Appl. Anal. Discrete Math. 12 (2018), no. 1, 153-165.

[31] F. Qi and B.-N. Guo, Explicit and recursive formulas, integral representations, and properties of the large Schröder numbers, Kragujevac J. Math. 41 (2017), no. 1, 121-141.

[32] F. Qi and B.-N. Guo, Explicit formulas for special values of the Bell polynomials of the second kind and for the Euler numbers and polynomials, Mediterr. J. Math. 14 (2017), no. 3, Article 140, 14 pages.

[33] F. Qi and B.-N. Guo, Expressing the generalized Fibonacci polynomials in terms of a tridiagonal determinant, Matematiche (Catania) 72 (2017), no. 1, 167-175.

[34] F. Qi and B.-N. Guo, Lévy-Khintchine representation of Toader-Qi mean, Math. Inequal. Appl. 21 (2018), no. 2, $421-431$.

[35] F. Qi and B.-N. Guo, Several explicit and recursive formulas for generalized Motzkin numbers, AIMS Math. 5 (2020), no. 2, 1333-1345.

[36] F. Qi and B.-N. Guo, Some determinantal expressions and recurrence relations of the Bernoulli polynomials, Mathematics 4 (2016), no. 4, Article 65, 11 pages.

[37] F. Qi and B.-N. Guo, The reciprocal of the geometric mean of many positive numbers is a Stieltjes transform, J. Comput. Appl. Math. 311 (2017), 165-170.

[38] F. Qi and B.-N. Guo, The reciprocal of the weighted geometric mean is a Stieltjes function, Bol. Soc. Mat. Mex. (3) 24 (2018), no. 1, 181-202.

[39] F. Qi and B.-N. Guo, The reciprocal of the weighted geometric mean of many positive numbers is a Stieltjes function, Quaest. Math. 41 (2018), no. 5, 653-664.

[40] F. Qi and B.-N. Guo, Two nice determinantal expressions and a recurrence relation for the Apostol-Bernoulli polynomials, J. Indones. Math. Soc. (MIHMI) 23 (2017), no. 1, 81-87.

[41] F. Qi, C. Kızllateş, and W.-S. Du, A closed formula for the Horadam polynomials in terms of a tridiagonal determinant, Symmetry 11 (2019), no. 6, 8 pages.

[42] F. Qi, O. Kouba, and I. Kaddoura, Computation of several Hessenberg determinants, Math. Slovaca 70 (2020), in press; OSF Preprints (2020), available online at https://doi.org/10.31219/osf.io/x4unj

[43] F. Qi and D. Lim, Integral representations of bivariate complex geometric mean and their applications, J. Comput. Appl. Math. 330 (2018), 41-58.

[44] F. Qi, M. Mahmoud, X.-T. Shi, and F.-F. Liu, Some properties of the Catalan-Qi function related to the Catalan numbers, SpringerPlus (2016), $\mathbf{5}: 1126,20$ pages.

[45] F. Qi, D.-W. Niu, and B.-N. Guo, Some identities for a sequence of unnamed polynomials connected with the Bell polynomials, Rev. R. Acad. Cienc. Exactas Fís. Nat. Ser. A Math. RACSAM 113 (2019), no. 2, 557-567.

[46] F. Qi, E. Polatli, and B.-N. Guo, Determinantal formulas and recurrent relations for bi-periodic Fibonacci and Lucas 
polynomials, OSF Preprints (2020), available online at https://doi.org/10.31219/osf.io/qvxd8

[47] F. Qi, X.-T. Shi, and B.-N. Guo, Integral representations of the large and little Schröder numbers, Indian J. Pure Appl Math. 49 (2018), no. 1, 23-38.

[48] F. Qi, X.-T. Shi, and B.-N. Guo, Some properties of the Schröder numbers, Indian J. Pure Appl. Math. 47 (2016), no. 4, $717-732$.

[49] F. Qi, X.-T. Shi, and B.-N. Guo, Two explicit formulas of the Schröder numbers, Integers 16 (2016), Paper No. A23, 15 pages.

[50] F. Qi, J.-L. Wang, and B.-N. Guo, A determinantal expression for the Fibonacci polynomials in terms of a tridiagonal determinant, Bull. Iranian Math. Soc. 45 (2019), no. 6, 1821-1829.

[51] F. Qi, J.-L. Wang, and B.-N. Guo, A representation for derangement numbers in terms of a tridiagonal determinant, Kragujevac J. Math. 42 (2018), no. 1, 7-14.

[52] F. Qi, X.-J. Zhang, and W.-H. Li, An elementary proof of the weighted geometric mean being a Bernstein function, Politehn. Univ. Bucharest Sci. Bull. Ser. A Appl. Math. Phys. 77 (2015), no. 1, 35-38.

[53] F. Qi, X.-J. Zhang, and W.-H. Li, An integral representation for the weighted geometric mean and its applications, Acta Math. Sin. (Engl. Ser.) 30 (2014), no. 1, 61-68.

[54] F. Qi, X.-J. Zhang, and W.-H. Li, Lévy-Khintchine representation of the geometric mean of many positive numbers and applications, Math. Inequal. Appl. 17 (2014), no. 2, 719-729.

[55] F. Qi, X.-J. Zhang, and W.-H. Li, Lévy-Khintchine representations of the weighted geometric mean and the logarithmic mean, Mediterr. J. Math. 11 (2014), no. 2, 315-327.

[56] F. Qi and J.-L. Zhao, Some properties of the Bernoulli numbers of the second kind and their generating function, Bull. Korean Math. Soc. 55 (2018), no. 6, 1909-1920.

[57] F. Qi, J.-L. Zhao, and B.-N. Guo, Closed forms for derangement numbers in terms of the Hessenberg determinants, Rev. R. Acad. Cienc. Exactas Fís. Nat. Ser. A Mat. RACSAM 112 (2018), no. 4, 933-944.

[58] Z.-H. Sun, A kind of orthogonal polynomials and related identities, J. Math. Anal. Appl. 456 (2017), no. 2, 912-926.

[59] Z.-W. Sun, Arithmetic properties of Delannoy numbers and Schröder numbers, J. Number Theory 183 (2018), $146-171$.

[60] Z.-W. Sun, On Delannoy numbers and Schröder numbers, J. Number Theory 131 (2011), no. 12, $2387-2397$.

[61] Z.-W. Sun, Supercongruences involving dual sequences, Finite Fields Appl. 46 (2017), 179-216.

[62] H. W. Turnbull, The Theory of Determinants, Matrices, and Invariants, 3rd ed., Dover Publications, New York, 1960.

[63] Y. Wang, S.-N. Zheng, and X. Chen, Analytic aspects of Delannoy numbers, Discrete Math. 342 (2019), no. 8, $2270-2277$.

[64] C.-F. Wei and F. Qi, Several closed expressions for the Euler numbers, J. Inequal. Appl. 2015, 2015:219, 8 pages. 